\title{
Ugandan Women's View of the IUD: Generally Favorable but Many Have Misperceptions About Health Risks
}

Rogers Twesigye, ${ }^{a}$ Peter Buyungo, ${ }^{a}$ Henry Kaula, ${ }^{a}$ Dennis Buwembo ${ }^{a}$

\begin{abstract}
Women in Uganda are aware of the IUD and think it is an effective method, but many think it can damage the womb or make a woman infertile. Addressing public misperceptions through interpersonal communication and the mass media, and provider misperceptions through training, can help to build demand for the IUD in Uganda.
\end{abstract}

\section{ABSTRACT}

Background: Between 2001 and 2006, IUD use in Uganda stagnated at $0.2 \%$ among women of reproductive age (WRA) ages 15-49. By 2011, IUD use had increased slightly to $0.4 \%$. Recent studies report a significant increase in IUD use to $3.8 \%$, but it is still low. Because the IUD is a little-used method in Uganda, we assessed the acceptability of the IUD by surveying women about their perceptions, attitudes, and beliefs.

Methods: In August and September 2014, we conducted a cross-sectional survey among 1,505 WRA exiting public and private health facilities in Uganda. We collected information on women's attitudes, knowledge, and beliefs about the IUD, as well as their perceptions about its availability. We classified women's responses according to a behavior change framework with 3 summary constructs: opportunity (structural factors that influence behavior), ability (skills to perform the behavior), and motivation (self-interest in adopting the behavior). As these 3 types of factors are more favorable to the desired behavior (in this case, use of the IUD), individuals are more likely to perform the behavior. Cross-tabulations compared the percentage results of perceptions and knowledge by key background characteristics.

Results: Most (87.8\%) of the surveyed women had heard of the IUD, and nearly two-thirds had a positive attitude toward the method. But a lower percentage (38.6\%) had accurate information about the IUD and more than half $(51.6 \%)$ did not think the method was available in a nearby facility. More than half of the women believed incorrectly that the IUD can damage the womb (57\%), that it reduces sexual pleasure (54\%), and that it can cause cancer (58\%). Current use of family planning or of a modern method specifically was positively associated with awareness and accurate knowledge and beliefs about the IUD. Married women had significantly higher awareness of the IUD than single women, and they had better knowledge and belief scores. The type of facility used for health care services (public, private franchise, or private non-franchise) may also influence acceptance of the IUD.

Conclusion: Interventions to increase the use of IUDs in Uganda should address low availability of the method in facilities, as well as misperceptions and misinformation, especially about the safety of the IUD. Demand promotion should address provider misperceptions in addition to client misperceptions and should include interpersonal communication and the mass media.

\section{BACKGROUND}

U ganda, with a population of 34.9 million, ${ }^{1}$ has one of the highest population growth rates in the world: $3.3 \%$ per year. ${ }^{2}$ Its total fertility rate of 5.8 children per woman in 2015 is also one of the highest in the world, despite a reduction from 6.2 children per woman in

\footnotetext{
a Programme for Accessible Communication and health Education (PACE), Kampala, Uganda.

Correspondence to Rogers Twesigye (rtwesigye@gmail.com).
}

$2011 .{ }^{3,4}$ About $42.6 \%$ of recent births were unintended: $27.7 \%$ of women wanted a child later, and $14.9 \%$ did not want another child. ${ }^{4}$

An almost-equal share of current contraceptive users in the country obtain their methods from the public and private sectors: $47 \%$ from public facilities, which provide free family planning services, and $45 \%$ from private providers. ${ }^{3}$ Public facilities tend to offer more contraceptive methods than private providers. For example, in 2015 39\% of public-sector facilities had 


\section{FIGURE 1. Modern Contraceptive Method Mix Among Married Contraceptive Users, Uganda,} 2015

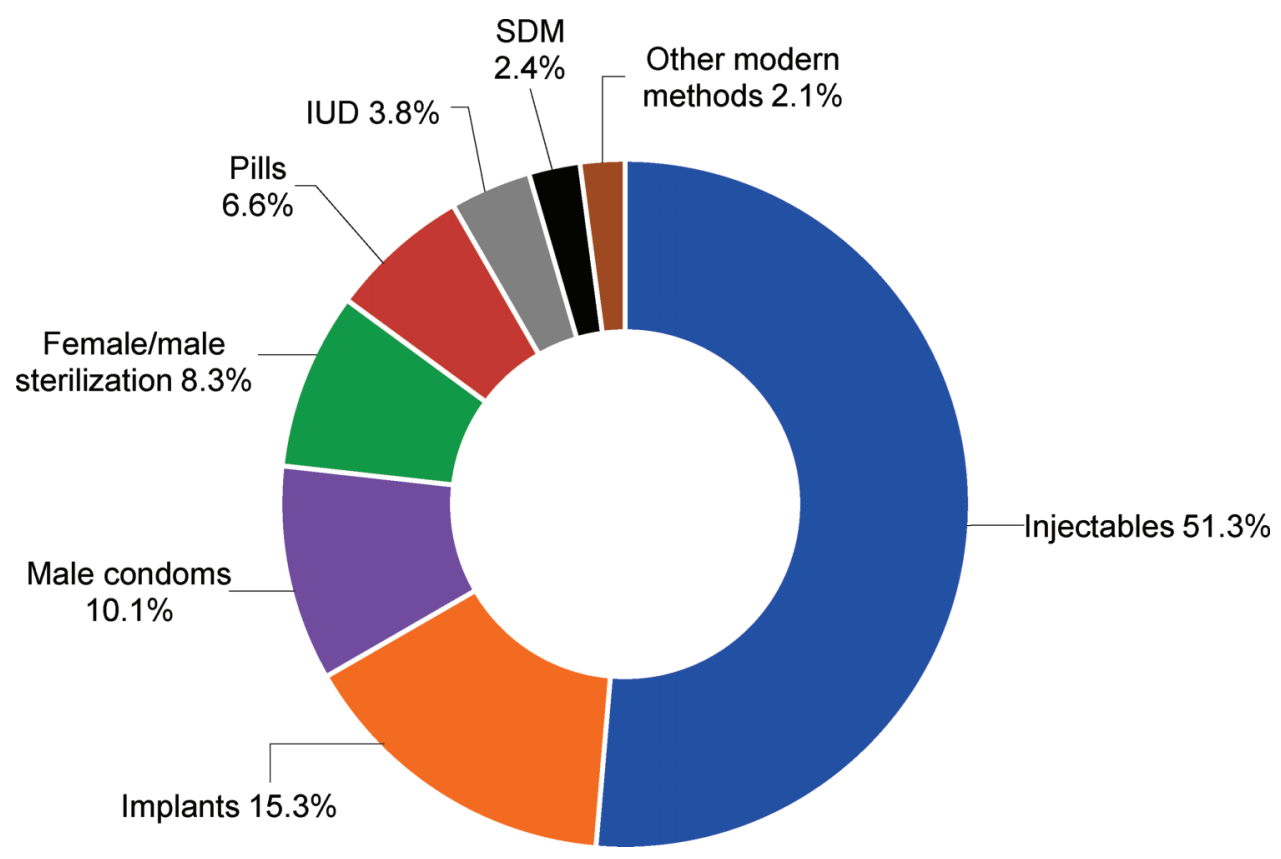

Abbreviations: IUD, intrauterine device; SDM, Standard Days Method.

Source: PMA, 2015-Uganda. ${ }^{3}$

IUDs in stock, compared with $7 \%$ of private providers, and $48 \%$ and $12 \%$, respectively, had implants in stock. ${ }^{4}$

In the past 5 years, the unmet need for family planning in Uganda decreased modestly from $34 \%$ of women of reproductive age (WRA) ages 15-49 in 2011 to $30 \%$ in 2015 . Use of modern contraceptive methods increased from $26 \%$ to $32 \%$ during the same period, and use of long-acting reversible contraceptives (LARCs)—comprising implants and the intrauterine device (IUD) -increased from 6\% to 9\% of total modern method use. ${ }^{4}$

Contraceptive prevalence in Uganda is dominated by injectables, male condoms, and other short-acting methods (Figure 1). Among married contraceptive users, the copper IUD makes up the smallest proportion of the current method mix at $3.8 \%$ compared with $6.6 \%$ for pills, $8.3 \%$ for sterilization (male and female), $10.1 \%$ for male condoms, $15.3 \%$ for implants, and $51.3 \%$ for injectables. ${ }^{4}$ Although the share of the IUD in the method mix is small, use of the method was only $0.4 \%$ in 2011 , and thus its use has increased dramatically.

Because IUDs are a little-used method in Uganda, we wanted to survey women's knowledge, attitudes, and beliefs about it. We used data from a survey conducted in 2014 among women ages $15-49$ years exiting public and private health facilities located in the area of the Women's Health Project (WHP). This project has been implemented since July 2008 by the Programme for Accessible health Communication and Education (PACE) or Population Services International (PSI) Uganda. A network of ProFam-branded health facilities and partner health facilities carries out WHP. By September 2014, the ProFam franchise had more than 195 facilities in 60 districts in all regions of the country.

WHP aims to increase demand for and access to a wide range of contraceptive methods, with a particular emphasis on increasing access to the IUD, mix at $3.8 \%$. 
FIGURE 2. Sampling Procedure: Participants Interviewed at Public, Private, and Pro-Fam Franchise Facilities in Each of the 38 Selected Subcounties

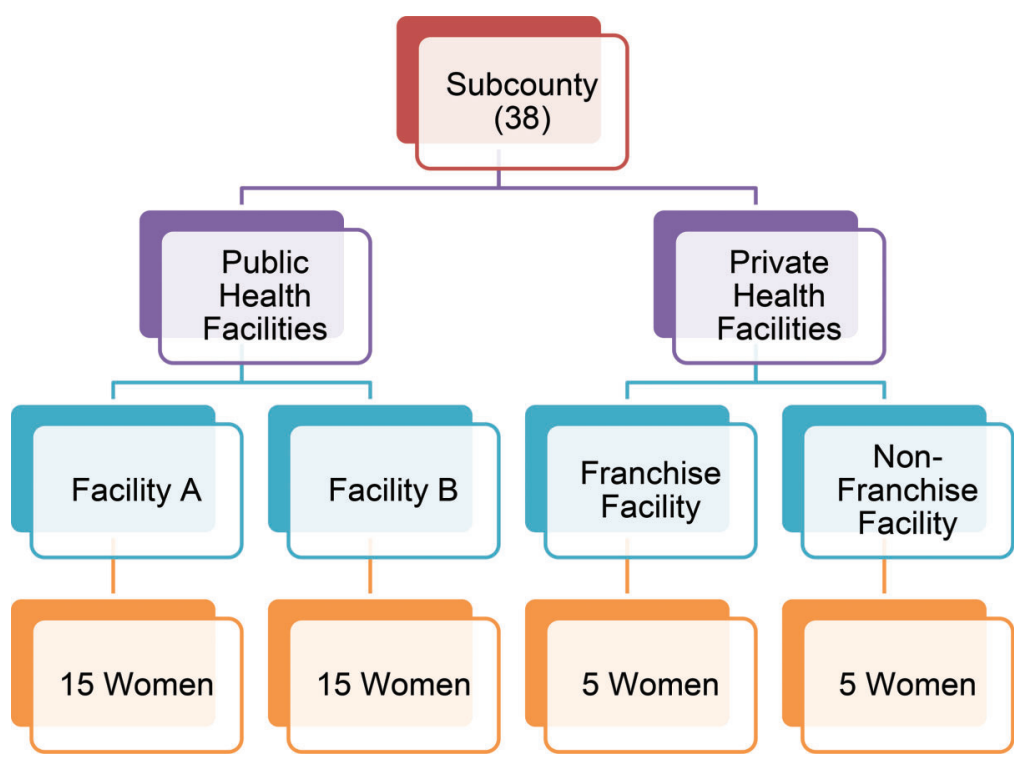

among WRA by improving knowledge and correcting misperceptions related to modern methods through interpersonal communication and the mass media. For interpersonal communication, community-based mobilizers are trained and commissioned to conduct door-to-door sensitization and education of WRA about LARCs and other family planning services. Mass media activities include branding and radio and television advertising. The program also trains providers to insert IUDs and provides insertion kits and subsidized IUDs to franchise facilities.

\section{METHODS}

\section{Design}

We carried out the survey in August and September 2014. We used a cross-sectional survey design with structured face-to-face exit interviews, and a multistage cluster sampling to select the study sites at the subcounty and facility level. We drew up a list of all subcounties in 60 project districts with their respective populations and used the probability proportional to population size approach to select 38 subcounties. Four health facilities2 public and 2 private-were selected in each sampled subcounty using simple random methods from lists of health facilities in the subcounty. For private facilities, we selected 1 franchise facility in the subcounty and 1 non-franchised but private facility (Figure 2).

\section{Selection of Survey Participants}

A total of 1,505 WRA were interviewed when leaving the selected health facilities, irrespective of the services they had received. Interviewers screened all females for eligibility whom they judged to be 15-49 years old. Each selected subcounty contributed 40 WRA toward the overall sample. Public health facilities contributed 30 client exit interviews in each subcounty15 interviews at each facility. The private health facilities contributed 10 client interviews - 5 interviews at the franchise facility and another 5 at the selected non-franchise facility. Analysis for correct knowledge, attitudes, and beliefs was only done among the $87.8 \%$ of participants $(n=1,321)$ who said that they had heard of the IUD, either spontaneously or after prompting.

\section{Study Instruments and Variables}

We collected information on demographics, access to media, fertility history, contraceptive knowledge, 


\section{The PSI behavior change framework has 3 summary constructs: opportunity, ability, and motivation.}

and perceptions about family planning in general and about IUDs in particular. We also looked at levels of exposure to communication messages about family planning.

Awareness and knowledge questions were measured using dichotomous variables, whereas perceptions were measured using an evaluation of statements on a 5-point Likert scale, from strongly disagree (1) to strongly agree (5). The questionnaire was designed in English (see supplementary material) and translated into the 6 main languages in the study area.

We classified perceptions according to a behavior change framework adopted by PSI based on a literature review of behavior change theories. This framework has 3 summary constructs: opportunity, ability, and motivation. ${ }^{5}$ Each of the summary constructs comprises different but related factors. As the opportunity, ability, and motivation factors are more favorable to the promoted behavior, individuals are more likely to perform the behavior. The following descriptions were adopted for IUD measurement from the PSI framework.

- Opportunity comprises external or structural factors that influence an individual's chance to perform a promoted behavior. The opportunity factors measured were awareness and availability. On perceived availability of IUDs, participants were asked to respond to statements such as "IUDs are available in facilities nearby in the community."

- Ability comprises an individual's skills or proficiencies to perform a promoted behavior. The factor measured under ability was knowledge-whether the individual had correct information about IUDs. Correct information was assessed by 3 statements to which participants had to answer true, false, or don't know: The IUD can be used while breastfeeding [correct response: true]; Once the IUD is removed, a woman can get pregnant [correct response: true]; and The IUD protects against sexually transmitted infections (STIs) [correct response: false].

- Motivation measures a woman's self-interest in adopting the IUD. Motivation was measured by looking at attitudes, beliefs, and outcome expectations. Attitudes refer to an individual's assessment of the promoted behavior. Items used to measure attitudes included: The IUD is a good method of family planning; The IUD is a safe family planning method; and The IUD is an effective family planning method. Women chose answers from the 5-point Likert scale. Beliefs are perceptions about a promoted behavior that may or may not be true. Items were measured on the 5-point Likert scale and included: IUDs can result in cancer; IUDs can damage the womb; and Prolonged use of IUDs can cause infertility.

\section{Data Analysis}

We used SPSS version 18 to analyze the data. Correct knowledge was computed by creating a new variable for participants who gave correct answers for all 3 knowledge questions. For the scaled responses, we conducted factor analysis to load similar statements together that resulted in 2 components-attitudes and beliefs/myths about the IUD. We used Cronbach's alpha to calculate internal reliability and consistency of the 2 created components (Table 1). Composite scores based on a scale of 1-5 were thereafter computed for each created component. A higher score related to a positive attitude (acceptable) and a lower score to a negative attitude (unacceptable). The reverse was true for the score on the second component related to myths about the IUD; a lower score in this case related to a positive attitude. Crosstabulations compared the percentage results of perceptions and knowledge by key population characteristics, and we conducted UNIANOVA analysis to compare mean scores for the 2 created components and key characteristics and to test the significance of observed differences.

\section{Ethical Procedures}

The study was approved by the Mildmay Uganda Research Ethics Committee (MUREC) and registered with the Uganda National Council for Science and Technology (UNCST).

\section{RESULTS}

\section{Characteristics of the Study Participants}

The majority $(85.9 \%)$ of the survey participants were 34 years or less, and $70.3 \%$ were either married or cohabiting (Table 2). Slightly over half $(52.3 \%)$ of the participants had either no education or only a primary education; the rest had a secondary education or higher. More than threequarters $(81.0 \%)$ were Christians $(32.1 \%$ Catholics, 29.7\% Protestant, and 19.2\% Pentecostal), and $18.9 \%$ were Muslims. Half $(50.8 \%)$ of the 


\begin{tabular}{lr}
\hline TABLE 1. Reliability Analysis Using Cronbach's Alpha for Attitudes and Beliefs About the IUD \\
\hline Attitudes & Cronbach's alpha \\
The IUD is a good method of family planning. & 0.862 \\
IUDs are safe. & \\
The IUD is an effective contraceptive method for women like me. \\
Beliefs \\
Using IUDs can result in cancer. \\
IUDs can damage the womb. \\
Prolonged use of IUDs can cause infertility.
\end{tabular}

Abbreviations: IUD, intrauterine device. participants reported that they regularly or at times read newspapers. A similar proportion regularly or at times watched television, whereas almost all (92.4\%) listened to the radio sometime every month.

More than three-quarters of the participants $(81.2 \%)$ had ever given birth in their lifetime (Table 2 ); $40.6 \%$ had given birth to 1 or 2 children, about one-quarter $(23.5 \%)$ had 3-4 children, and $17.1 \%$ had given birth to 5 or more children. Just over half $(53.0 \%)$ of the participants (exclusive of those who were pregnant) were using some form of contraception to delay or avoid pregnancy, and $44.1 \%$ were using a modern contraceptive method.

\section{Opportunity: Awareness of the IUD}

The majority $(87.8 \%)$ of the participants had heard of the IUD as a contraceptive method. Awareness of the IUD varied by marital status, age group, current use of family planning, and TV viewership (Table 3). For example, significantly more married women had heard of the IUD than unmarried women (90.8 vs. $80.5 \%$, respectively; $P<.001)$. Similarly, significantly more women using any form of contraception had ever heard of the IUD than those not using a contraceptive $(91.5 \%$ vs. $82.5 \%$, respectively; $P<.001)$. The trend was similar for women using modern contraceptives in comparison with those using traditional methods $(92.6 \%$ vs. $86.1 \%$, respectively; $P=.001$ ). No statistically significant differences were observed in awareness of the
IUD and parity, whether one listens to the radio, or the type of facility used for health care services.

Opportunity: Perceived Availability of IUDs Overall, $48.4 \%$ of participants perceived IUDs as available in a facility nearby in the community. Perceived availability of IUDs varied by use of modern contraception, age group, and source of health services. Most notably, significantly more women using modern contraceptives than those using traditional methods perceived IUDs as available in a nearby facility $(50.3 \%$ vs. $43.3 \%$, respectively; $P=.05$ ). No statistically significant differences were observed on perceived availability of IUDs and marital status, parity, use of any family planning method, radio listenership, or TV viewership.

\section{Ability: Correct Knowledge About IUDs}

Overall, $38.6 \%$ of the women who had heard of the IUD knew that it can be used during breastfeeding, that a woman can get pregnant once the IUD is removed, and that the IUD does not protect against STIs. Correct information about the IUD varied by marital status, age group, current use of family planning, and radio listenership (Table 3). No statistically significant differences were observed in correct knowledge about IUDs and parity, TV viewership, or the type of facility used for health care services. 


Nearly two-thirds
of surveyed
women believed
that IUDs are safe,
but many
surveyed
women had
misconceptions
about the method.

TABLE 2. Characteristics of Survey Participants $(\mathrm{N}=1,505)$

\begin{tabular}{lc}
\hline Characteristic & $\%$ \\
\hline Age group & \\
$15-24$ & 48.2 \\
$25-34$ & 37.7 \\
$35-49$ & 14.1
\end{tabular}

Marital status

Not married

Married/cohabiting

70.3

Education

No formal education

Primary

Secondary

Above secondary

Religion

Catholic

Protestant

Pentecostal

Muslim

44.6

38.5

Access to media

Reads newspapers

50.8

Listens to radio

Watches television

Parity

Ever given birth

Never given birth

No. of living children

0

$1-2$

18.8

40.6

3-4

23.5

5 or more

Contraceptive use

Any method

Modern method

\section{Motivation: Attitude Toward IUDs}

The study results showed that nearly two-thirds of the women believed that IUDs are safe (60.3\%) and are a good method of family planning $(63.7 \%)$. In addition, $59.0 \%$ reported that IUDs are an effective family planning method, while a notable percentage $(45.8 \%)$ would recommend an IUD to a friend. Women scored the IUD above average (3.6 out of 5) as a good, safe, and effective family planning method (Table 4). No background characteristic showed significant variations on attitude toward the IUD.

\section{Motivation: Beliefs About IUDs}

Overall, women seemed to agree with or were not sure about the incorrect beliefs about the IUD. More than half $(57 \%)$ of the women believed IUDs can damage the womb and a similar percentage (54\%) thought IUDs reduce sexual pleasure. More than half $(58 \%)$ also believed that the IUD can cause cancer while close to half $(48 \%)$ believed that prolonged IUD use can cause infertility. On average, women scored the IUD 3.6 out of 5 in relation to causing cancer, damaging the womb, and causing infertility.

Beliefs about IUDs were found to vary by marital status, age group, current use of family planning, and source of health services (Table 4). Married women, for example, scored significantly lower than unmarried women on the incorrect beliefs about IUDs $(3.0$ vs. $3.5 ; P<.001)$. This implies that married women refuted incorrect beliefs about IUDs more than their counterparts and therefore were more likely to accept the IUD. Notably, women at the project franchise facilities were more accepting of the IUD compared with those at public and other private facilities. No statistically significant differences were observed in incorrect beliefs and number of children, use of modern contraceptives, and TV viewership or radio listenership.

\section{DISCUSSION}

Despite the low use of the IUD in Uganda, a high percentage of women surveyed in this studynearly $90 \%$-have heard of the IUD. By comparison, in the 2011 Uganda Demographic and Health Survey, $70.2 \%$ of women said that they had heard of the IUD. ${ }^{3}$ The surveyed women in this study generally have a positive attitude toward the IUD as a safe and effective family planning method, but the majority have inaccurate knowledge about the method, for example, about return 
TABLE 3. Opportunity and Ability Factors (\%) Related to IUDs Among Survey Participants by Background Characteristics

\begin{tabular}{|c|c|c|c|c|c|c|}
\hline \multirow[b]{2}{*}{ Characteristic } & \multicolumn{4}{|c|}{ Opportunity } & \multicolumn{2}{|c|}{ Ability } \\
\hline & $\begin{array}{c}\text { Heard } \\
\text { of IUDs } \\
(\mathrm{N}=1,505)\end{array}$ & P Value & $\begin{array}{l}\text { Perception of IUD } \\
\text { Availability } \\
(n=1,321)^{a}\end{array}$ & P Value & $\begin{array}{l}\text { Correct IUD } \\
\text { Knowledge } \\
(n=1,321)^{b}\end{array}$ & P Value \\
\hline Marital status & & $<.001$ & & .45 & & .005 \\
\hline Married/cohabiting & 90.8 & & 49.0 & & 40.9 & \\
\hline Not married & 80.5 & & 46.7 & & 32.5 & \\
\hline Age & & $<.001$ & & .02 & & .01 \\
\hline $15-24$ & 84.0 & & 45.0 & & 34.4 & \\
\hline $25-34$ & 92.0 & & 53.0 & & 41.8 & \\
\hline $35-49$ & 89.2 & & 46.0 & & 43.8 & \\
\hline Parity & & .92 & & .85 & & .07 \\
\hline $1-2$ & 90.5 & & 48.8 & & 37.4 & \\
\hline $3-4$ & 90.9 & & 50.8 & & 41.7 & \\
\hline 5 or more & 89.9 & & 50.0 & & 46.1 & \\
\hline Uses family planning & & $<.001$ & & .97 & & .006 \\
\hline No & 82.5 & & 48.9 & & 33.7 & \\
\hline Yes & 91.5 & & 49.0 & & 41.9 & \\
\hline Type of family planning & & .001 & & .05 & & .03 \\
\hline Traditional & 86.1 & & 43.3 & & 35.6 & \\
\hline Modern & 92.6 & & 50.3 & & 43.3 & \\
\hline Listens to radio & & .13 & & .30 & & .006 \\
\hline Not at all & 85.3 & & 72.5 & & 25.3 & \\
\hline Usually & 88.1 & & 78.7 & & 39.6 & \\
\hline Watches TV & & .01 & & .70 & & .83 \\
\hline Not at all & 85.3 & & 77.7 & & 38.9 & \\
\hline Usually & 89.7 & & 78.8 & & 38.4 & \\
\hline $\begin{array}{l}\text { Type of facility used for } \\
\text { health care services }\end{array}$ & & .29 & & .008 & & .30 \\
\hline Public & 86.5 & & 48.6 & & 36.5 & \\
\hline Private, non-franchise & 89.5 & & 45.6 & & 40.3 & \\
\hline Private, franchise & 87.9 & & 58.6 & & 41.4 & \\
\hline Total & 87.8 & & 48.4 & & 38.6 & \\
\hline
\end{tabular}

Abbreviation: IUD, intrauterine device.

Significant $P$ values $\leq .05$ are shown in boldface.

a Percentage of women who had heard of IUDs and who responded positively to the statement, "IUDs are always available in the community and in a facility nearby."

b Percentage of women who had heard of IUDs and who knew the correct answers to the following statements: "IUDs can be used while breastfeeding" (correct answer: yes); "Once removed, a woman can get pregnant" (correct answer: yes); and "UDs protect against sexually transmitted infections" (correct answer: no). 
TABLE 4. Motivation Factors Related to IUDs Among Survey Participants by Background Characteristics $(N=1,505)$

\begin{tabular}{lcccc}
\hline Characteristic & Mean Attitude Score $^{\mathbf{a}}$ & P Value & Mean Beliefs Score $^{\mathbf{b}}$ & P Value \\
\hline Marital status & & .62 & & $<.001$ \\
Married/cohabiting & 3.6 & & 3.0 & \\
Not married & 3.6 & & 3.5 & \\
Age group & & .09 & & .04
\end{tabular}

Age group

$25-34$

$35-49$

Parity

$1-2$

3-4

5 or more

3.6

3.6

3.7

3.0

3.4

.64

Uses family planning

.35

No

3.4

Yes

3.6

3.0

Type of family planning

.44

Traditional

Modern

.48

Not at all

Usually

3.6

Watches TV .71

Not at all

Usually

3.6

3.1

Facility type

.25

Public

3.6

3.2

Private, non-franchise

3.5

3.3

Private, franchise

2.8

Overall score

3.6

Abbreviation: IUD, intrauterine device.

Significant $P$ values $\leq .05$ are shown in boldface.

a Women's scores out of a total 5 for the following 3 statements: The IUD is a good family planning method; The IUD is a safe family planning method; and The IUD is an effective family planning method.

b Women's scores out of a total 5 for the following 3 statements: The IUD can result in cancer; The IUD can damage the womb; and Prolonged use of IUDs can cause infertility. 
to fertility after IUD removal, and the majority also believe in myths surrounding the IUD. For example, over $40 \%$ of the participants had incorrect information about the IUD in relation to breastfeeding, return to fertility, and protection against STIs. Participants also believed incorrectly that IUDs cause cancer and damage the womb.

Availability of IUDs is limited by the lack of skilled providers and the lack of equipment. Over half of the survey respondents perceived a lack of availability of IUDs in nearby facilities. This perception is supported by findings from other surveys. For example, in a 2014 survey of privatesector providers, over $70 \%$ of the providers had ever had training in IUD insertion, but only $4 \%$ of franchise providers and 13\% of non-franchise providers had comprehensive training, including lectures, practice on models, and performing an insertion and removal on a patient under observation. ${ }^{6}$ Furthermore, only $30 \%$ of the providers correctly identified 3 of 5 side effects of the IUD. ${ }^{6}$ Providers are a key source of information for women seeking family planning, and a knowledge gap on their side results in a knowledge gap among women, which affects acceptance of a particular family planning method.

In our study, several factors emerged as having an influence on awareness, knowledge, and beliefs about the IUD. Current use of any family planning method or of a modern method specifically was positively associated with awareness and accurate knowledge and beliefs about the IUD. Women using contraceptives are more likely to be exposed to information about the IUD at a facility or from a health care provider than nonusers. In addition, married women had significantly higher awareness of the IUD than single women and had better knowledge and belief scores. This might be attributed to the fact that married women have already had children and therefore are in need of limiting births or spacing for a longer period. TV viewership seemed to play a more significant role in improving awareness about the IUD, whereas radio played a more significant role in women having correct knowledge about the method. Finally, the type of facility used for health care services may also influence acceptance of the IUD as a contraceptive method. Women at franchise facilities believed less in IUD-related myths than women at public facilities or private but non-franchise clinics. Women at franchise clinics also perceived the IUD as being available in the community. This might be attributed to the efforts to increase
IUD use by PACE through its Women's Health Project.

\section{Study Limitations}

Conducting the study in project implementation areas may have led to some bias since the ProFam franchise is in only 60 of 112 districts. However, we believe that this bias was reduced by the nationwide promotion of the IUD and the use of a random sample of non-franchise facilities and participants. Although women were selected randomly, only WRA who visited facilities on days when research teams were visiting had the chance to participate in the study. The study may also suffer from a seasonality effect since data were collected over a short period of time.

\section{CONCLUSION}

Use of the IUD in Uganda is increasing rapidly but is still low. To further increase access to and use of the IUD, couples need accurate information about the benefits and risks of the IUD. This information can come through interpersonal communication, from trained providers, and in the mass media. To protect women's health in Uganda, promoting demand for this safe and effective contraceptive method, in the context of wide method choice, should be a priority.

Acknowledgments: We would like to acknowledge support from Women's Health Project, Programme for Accessible health Communication and Education (PACE), and Population Services International.

Competing Interests: None declared.

\section{REFERENCES}

1. Uganda Bureau of Statistics (UBOS). National population and housing census 2014: provisional results. Kampala (Uganda): UBOS; 2014. Available from: http://www.ubos.org/onlinefiles/ uploads/ubos/NPHC/NPHC\%202014\%2OPROVISIONAL\% 20RESULTS\%20REPORT.pdf

2. The World Bank [Internet]. Washington (DC): The World Bank; c2016. Population growth (annual \%); [cited 2016 Jul 26]. Available from: http://data.worldbank.org/indicator/SP.POP. GROW

3. Uganda Bureau of Statistics (UBOS); ICF International. Uganda demographic and health survey 2011. Kampala (Uganda): UBOS; 2012. Co-published by ICF International. Available from: https://dhsprogram.com/pubs/pdf/FR264/ FR264.pdf

4. Performance, Monitoring and Accountability 2020 (PMA2020). PMA2015/Uganda-R2. Baltimore (MD): Johns Hopkins Bloomberg School of Public Health, PMA2020; 2015. Available from: http://www.pma2020.org/sites/default/files/UGR22PG-FPBrief-v7-2015-07.18.pdf

\section{Over half of the surveyed women did not think the IUD was available in a nearby facility.}

To protect women's health and slow Uganda's rapid population growth rate, promoting demand for the IUD, in the context of wide method choice, should be a priority. 
5. Population Services International (PSI). Concept paper: PSI behavior change framework. "Bubbles": proposed revision. Washington (DC): PSI; 2004. Available from: http://www.psi. org/wp-content/uploads/drupal/sites/default/files/ publication_files/behaviorchange.pdf
6. Population Services International-Uganda (PSI-Uganda). Health providers' knowledge, perceptions and practices related to long-term methods of family planning and post abortion care (PAC). TRaC Summary Report. Kampala (Uganda): PSI-Uganda; 2014.

\section{Peer Reviewed}

Received: 2015 Sep 30; Accepted: 2016 Mar 11

Cite this article as: Twesigye $R$, Buyungo $P$, Kaula $H$, Buwembo D. Ugandan women's view of the IUD: generally favorable but many have misperceptions about health risks. Glob Health Sci Pract. 2016;4 Suppl 2: S73-S82. http://dx.doi.org/10.9745/GHSP-D-15-00304.

(c) Twesigye et al. This is an open-access article distributed under the terms of the Creative Commons Attribution License, which permits unrestricted use, distribution, and reproduction in any medium, provided the original author and source are properly cited. To view a copy of the license, visit $\mathrm{http}: / /$ creativecommons.org/licenses/by/3.0/. When linking to this article, please use the following permanent link: http://dx.doi.org/

10.9745/GHSP-D-15-00304. 